\title{
El desarrollo de la sociología en el Perú. Notas introductorias
}

JULIO MEJÍA NAVARRETE

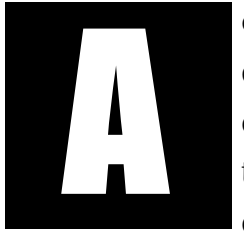

ctualmente se vienen dando profundas transformaciones que afectan a la sociedad y a los modos de construcción del pensamiento. Momento que manifiesta, de manera transparente, las grandes inquietudes que atraviesan y definen la construcción de la sociología. Precisamente, el estudio del desarrollo de la sociología en el Perú permite dar cuenta de las modificaciones que vienen ocurriendo en la realidad social y en sus procesos de conocimiento y explicación.

Sin embargo, no se trata de presentar únicamente una evolución de las principales ideas sociales en el Perú, sino que el artículo apunta a subrayar el carácter reflexivo de la sociología, en que medida su presencia y desarrollo ha influido en la sociedad. Ello implica que el mundo social no es sólo una estructura definida, sino también es producto y componente de una dinámica de estructuración del propio conocimiento.

El trabajo, de manera muy inicial, en un estudio preliminar intenta en forma sistemática reconstruir las diferentes etapas y enfoques de la sociología desde su creación en 1896 como cátedra universitaria en la

\footnotetext{
* Dr. en Ciencia Política y Sociología. Director de la Unidad de Post-Grado de la Facultad de Ciencias Sociales de la Universidad Nacional Myor de San Marcos (Perú). Docente de Sociología.
} 
Universidad de San Marcos. Presentar una visión de conjunto de la evolución de la sociología, supone, por un lado, hilvanar un discurso que permita establecer una tendencia, el de los avatares del desarrollo de modernidad en nuestro país, y, al mismo tiempo, significa apoyarse en el debate teórico generado en el proceso de constitución de la sociología, del lento proceso de institucionalización y en la toma de una mayor conciencia, en los últimos años, acerca de la pluralidad de paradigmas, perspectivas metodológicas y posibilidades interpretativas de la realidad social.

Esta alternativa favorece a la definición de algunos aspectos esenciales, prolongar el debate y abrir discusiones de los problemas que atañen, en general, al desarrollo de las ciencias sociales en el Perú.

\section{El pensamiento social peruano}

La fundación de un pensamiento social moderno en el Perú, que se desarrolla desde fines del siglo XIX y perdura hasta los años cincuenta del siglo XX, coincide con la creación de la Cátedra de Sociología en la Universidad Nacional Mayor de San Marcos en 1896. Sin embargo, la cátedra de sociología sólo significó un hecho subsidiario en el vasto debate de ideas generado en ese momento.

El establecimiento de un pensamiento social es la etapa clásica de las ideas sociales en el Perú, por el aporte a la comprensión de la sociedad que realizara la denominada generación del novecientos, por acercarse al conocimiento de la realidad nacional y formular alternativas para su desarrollo. Desde posiciones diferentes destacan Francisco García Calderón, José de la Riva Agüero, Víctor Andrés Belaunde, José Carlos Mariátegui, Víctor Raúl Haya de la Torre y Jorge Basadre, en la actualidad todavía son leídos y admirados en las escuelas de sociología, aunque no en la misma intensidad. 
Durante la segunda mitad del siglo XIX predominaban dos corrientes en el pensamiento social peruano: el positivismo en filosofía y el biologicismo en las ideas sociales. El positivismo significó cierto afán científico por estudiar la realidad, observar y acercarse a los problemas más urgentes del país, que implicaba el abandono de la tradición intelectualista y la sustitución por una perspectiva que enfatizaba en la modernización del país. ${ }^{1}$ En cambio, el biologicismo representaba la herencia colonial que explicaba la organización social a partir de las premisas genéticas y de una superioridad y dominio natural de las élites criollas. Clemente Palma es el mayor exponente de las ideas racistas, en 1897 publicó El porvenir de las razas en el Perú.

Esta situación cambia radicalmente al finalizar la guerra con Chile, el Perú se encontraba en una crisis sin precedentes, su capacidad económica había sido destruida, estaba políticamente desarticulado y moralmente disminuido. Para los primeros años del siglo XX entramos nuevamente en un proceso de reorganización de la sociedad, esta vez bajo la hegemonía del capitalismo monopolista y del desarrollo de una economía de enclave. La fundación de un pensamiento social en el Perú responde al desarrollo de un proyecto de la modernidad, que de alguna manera ha significado el tipo de sociedad que se construyó durante el siglo XX. Propuesta que haría eco de la tradición de las ciencias sociales, en la medida que cuanto mejor se conoce la realidad social mejores son las capacidades de modificarla, iniciada por Montesquieu y desarrollada por Comte y Durkheim, que luego ha tenido una gran vigencia. ${ }^{2}$

El pensamiento social moderno peruano se desarrolla como parte de la polémica entre el positivismo y el biologicismo. Aunque no todos tuvieron posiciones claras al respecto, esbozaron las primeras explicaciones integrales

\footnotetext{
1 Aníbal Quijano: Imperialismo, clases sociales y Estado en el Perú: 1890-1930, Mosca Azul, Lima, 1978, p. 83-84. 2 Anthony Giddens: En defensa de la sociología, Alianza Editorial, Madrid, 2000, p. 31.
} 
de la sociedad y política en el país. En ese sentido se establecieron los fundamentos del análisis sociológico de la realidad nacional del Perú; sin embargo, todavía en gran parte se encontraban enlazados a una tradición caracterizada por una reflexión especulativa y ensayística. En ese contexto se desarrollan principalmente dos perspectivas.

La primera perspectiva, más conservadora, desarrolla una filiación positivista liberal y representa las expresiones más modernizantes de las élites peruanas. Destacan Francisco García Calderón y Víctor Andrés Belaunde. El Perú contemporáneo (1907) de García Calderón es el iniciador del género de "estudio integral" sobre la realidad nacional. En este libro se analizan interrelacionadamente la historia, la coyuntura, las ideas, la economía, la política, la educación, la situación internacional y el porvenir. Por todo ello, se constituyó en el modelo de investigación de la realidad social del país para la generación del novecientos. En ella se formula un diagnóstico dualista de la sociedad peruana signado por la diferencia y contraste radical entre la costa y la sierra. La costa moderna, civilizada y sede de las élites criollas, mientras que la sierra representaba el atraso, el aislamiento y el reducto de la población indígena. Como proyecto se plantea el desarrollo de una oligarquía ilustrada, cohesionada y modernizante capaz de organizar e integrar la sociedad mediante la centralización de estatal. No desconoce los aspectos económicos ni de la tierra del problema indígena, pero destaca el papel de la difusión de nuevas tecnologías, la integración al mercado y la educación. Asume una perspectiva eurocéntrica que define las características y trayectoria europea como superior o mejor, mientras que lo nativo es visto como algo inferior y carente de valor. El paradigma racista sirve para argumentar una visión jerarquizada del indio en la sociedad, que por su condición requiere de un tutelaje transitorio y de la responsabilidad de las élites criollas. Sin embargo, donde la ideología racista se muestra con mayor plenitud, es cuando reconoce que el indio 
está condenado a desaparecer, en tanto ser racial y expresión del pasado colonial, por medio del mestizaje y cruce de razas. Aquí el mestizaje se muestra como una ideología basada en la premisa de la superioridad blanca del criollo y el desprecio a todo lo indígena que debe desaparecer. ${ }^{3}$

La perspectiva más crítica del orden oligárquico tiene entre sus exponentes mayores a Manuel González Prada, Víctor Raúl Haya de la Torre y José Carlos Mariátegui, quienes desarrollan verdaderas contribuciones originales y sugerentes al pensamiento social peruano. González Prada inicia una crítica radical al racismo y a la dominación oligárquica, recogiendo principalmente una predica moralista, y plantea la necesidad de reorganizar el orden social por medio de la integración social de la población indígena. Asimismo, desarrolla un sentimiento anticriollo de regeneración de la sociedad peruana. Sin embargo, es la polémica Mariátegui-Haya, desde una perspectiva marxista, que se convierte en el centro de la discusión en los años veinte y el fundamento del pensamiento crítico hasta los años sesenta. Buscan la explicación de las especificidades peruanas en la historia, la estructura social y la cultura nacional. Víctor Raúl Haya de la Torre formula un proyecto de la modernidad original de acuerdo a las singularidades de la sociedad peruana, basándose en las posiciones dualistas formula, de un lado, la necesidad de la autonomía de la clase burguesa y, de otro, el desplazamiento de la oligarquía, dentro de un proyecto encaminado al desarrollo de un capitalismo nacional por medio de un "Estado antiimperialista".

José Carlos Mariátegui, de modo particular en Siete ensayos de interpretación de la realidad peruana (1928), ofrece una perspectiva más analítica y científica de la sociedad peruana que permitió establecer bases

\footnotetext{
3 Catherine Walsh: "La (re)articulación de subjetividades políticas y diferencia colonial en el Ecuador. Reflexiones sobre el capitalismo y las geopolíticas del conocimiento", en Indisciplinar las ciencias sociales. Geopolíticas del conocimiento y colonialidad del poder. Perspectiva desde lo andino, Universidad Andina Simón Bolívar y Ediciones ABYA-YALA, Quito, 2000, p. 183-184.
} 
firmes para una reflexión sociológica. Mariátegui considera que las teorías no "constituyen principios de consecuencias rígidas", más bien adquieren un contenido concreto en el estudio de la realidad nacional. Es importante señalar que desarrolla un diagnóstico de la sociedad peruana como una totalidad heterogénea, que constituye uno de los aportes más importantes y originales de su pensamiento, integra lo moderno y no moderno, recoge las múltiples combinaciones de formas históricas diferentes que coexisten y se articulan en un mismo espacio-tiempo: feudal, capitalista, formas socializantes y hasta elementos esclavistas, las cuales no se encuentran en oposición y menos aún se suceden unas a otras en un desarrollo lineal, más bien el sentido y unidad del sistema se define por la lógica del capital. Dicho estudio le permitió plantear una propuesta que no sea "ni calco ni copia" de otras realidades sino la búsqueda de un "socialismo indoamericano".

El pensamiento social moderno que se desarrolla en los años veinte, sobre el carácter de la dependencia, el Estado y las fuerzas sociales, fue el centro de la discusión en el Perú y América Latina. Es el primer momento de una reflexión y contribución propia al desarrollo de las ideas latinoamericanas. Originalidad de propuestas que permitió tener vigencia hasta los años sesenta en la intelectualidad peruana. Cabe anotar que prácticamente los estudios sobre la realidad social desaparecieron desde 1930, se cayó en un período de esclerosis y paralización del pensamiento social hasta fines de los años cincuenta, como resultado de un momento de oscurantismo impuesto por los regímenes dictatoriales oligárquico-militares y del desarrollo de un proceso de dogmatización y repetición empobrecida de las ideas originales.

\section{La sociología como cátedra}


La sociología inicia su proceso de institucionalización con la creación en la Universidad Nacional Mayor de San Marcos de la cátedra en 1896 como parte del desarrollo de una conciencia social moderna en el Perú. Mariano H. Cornejo es nombrado el primer profesor de sociología, Carlos Wiesse (lo asume en 1908) y Roberto Mac Lean y Estenós (le sigue desde 1928 hasta 1953) son los responsables de regentar la Cátedra universitaria hasta los años cincuenta y los que llevan un producto intelectual exótico a la universidad, que por su naturaleza le impone serias limitaciones a la propia disciplina.

Nuestros catedráticos llegan a ser sociólogos de manera episódica, sólo cuando enseñan en la universidad, su formación y vocación no es la sociología, no están interesados en estudiar la realidad nacional. Cornejo es abogado, diplomático y político parlamentario. Wiesse destacó en la diplomacia y el periodismo. Mac Lean es abogado, político y parlamentario. La sociología es una preocupación y actividad secundaria, como lo es en el pensamiento social peruano.

En efecto, la expresión intelectual más conservadora y civilista de la generación del novecientos acapara intelectualmente la Universidad de San Marcos. La cátedra de sociología es parte de esas tendencias oligárquicas y clientelísticas que reproduce la rutina burocrática, cuando no ocurre sufre la intervención y la clausura. Por lo general, el desarrollo de la sociología en la universidad queda relegada a algún curso de relativo interés.

Mariano H. Cornejo, bajo la influencia de Herbert Spencer, publica Sociología General en dos tomos (1908 y 1910), donde expone de manera sistemática el pensamiento sociológico europeo. Esta obra de Cornejo marca el carácter de la sociología de este período como una reflexión fundamentalmente especulativa, sin ninguna intención de desarrollar la investigación empírica de la realidad peruana, preocupada más por exponer interpretaciones teorizantes sobre la sociedad en general y muy dependiente de la discusión europea. Carlos Wiesse, que sucede a Cornejo 
en la enseñanza, apuntaba a desarrollar el positivismo de Comte y Spencer, publicó Extractos de sociología, en dos volúmenes (1908 y 1909) donde sólo se limita a compilar un conjunto de lecturas con fines pedagógicos. Cornejo y Wiesse adoptan intelectualmente posiciones cercanas al positivismo conservador y en términos ideológicos son liberales moderados. Por su parte, Roberto Mac Lean publicó Sociología (1930), Sociología peruana (1942), Sociología educacional del Perú (1944) y Sociología integral (1951), libros que mantienen el plan inicial de la obra de Cornejo; en términos políticos más bien derivó sus ideas a favor del fascismo y de posiciones cercanas a una moral convencional tradicionalista. ${ }^{4}$

En ese sentido, la sociología asume un carácter eurocéntrico, lo moderno en el Perú es sinónimo de lo extranjero, el predominio de la innovación y novedad del conocimiento provienen inicialmente de la producción bibliográfica europea, a partir de la cual se define una visión que considera el desarrollo en forma lineal, siguiendo un orden de etapas ascendentes según el modelo occidental. El eurocentrismo consiste en la forma de comprender la realidad de América Latina según las características y desarrollo particular de Europa, se explica la realidad de nuestro continente a partir de categorías que fueron elaboradas para dar cuenta el mundo europeo, concepción que se transforma en una visión de alcance y validez universal.

En suma, nuestros primeros sociólogos desarrollan un proyecto de la modernidad de manera marginal, resultado subsidiario del debate de las ideas que se generó con fuerza en los primeros veinte años del siglo XX. La reflexión sociológica se inspira en las ideas positivistas, que intenta superar las posiciones metafísicas y religiosas del pasado, al no desarrollar la investigación como forma principal de acercamiento a la realidad nacional, se queda en un plano especulativo, general y eurocéntrico.

4 Guillermo Rochabrún: “Sociología y sociedad en el Perú: un esbozo histórico”, en Estudios de historia de la ciencia en el Perú, CONCYTEC - SOPHICYT, Lima, 1986, V. II, p. 162-163. 


\section{La institucionalización de la sociología}

La sociología se institucionaliza como carrera profesional en el Perú cuando en 1956 se funda el Instituto de Sociología en la Universidad Nacional Mayor de San Marcos. Asimismo, en la década del sesenta se crean casi simultáneamente las principales escuelas en el país, ${ }^{5}$ etapa que se caracteriza por la tensión constitutiva entre dos modelos de hacer sociología: son los reflejos iniciales de algún estructural-funcionalismo y marxismo que, por otro lado, es el rasgo esencial que define la sociología del siglo XX. Este momento se cierra con la instauración del gobierno militar de Velasco Alvarado en 1968.

La fundación de la carrera de sociología en el Perú giró en relación a dos propuestas centrales. ${ }^{6}$ La primera, sobre una sociología tecnológica con la intención de desarrollar profesionales "capacitados para resolver los problemas sociales", con apoyo de la UNESCO se creó en 1961 el Departamento de Sociología al interior de la Facultad de Letras en la Universidad de San Marcos y, posteriormente, con el auspicio del gobierno holandés se crea la Facultad de Ciencias Sociales en la Universidad Católica.

La segunda corriente, acerca de una sociología crítica, fue planteada tempranamente por Aníbal Quijano en Imagen y tareas del sociólogo en la sociedad peruana (1965) cuyo objetivo central de la investigación social es "convertir en problemática teórica las preocupaciones de las clases explotadas y la búsqueda de las vías para una transformación radical de la sociedad".

François Bourricaud, discípulo de Parsons, a partir de 1956 organizó los cursos y seminarios que serán los antecedentes para la fundación de la

\footnotetext{
5 Se crean las escuelas de sociología en la Universidad Nacional del Centro y Universidad Nacional Mayor de San Marcos, en 1961; en la Universidad Nacional de San Agustín de Arequipa, en 1963; en la Universidad Pontificia Católica del Perú, en 1964; y en la Universidad Nacional Federico Villarreal, en 1968.

6 César Germaná: La racionalidad en las ciencias sociales, Fondo Editorial de la Facultad de Ciencias Sociales, UNMSM, Lima, 2002, p.98.
} 
carrera de sociología en la Universidad de San Marcos. La formación inicial de la sociología en el Perú tuvo una influencia del estructural-funcionalismo en los aspectos teóricos, mientras que en la metodología se desarrollaba una concepción positivista y cuantitativa. La sociología incidía principalmente en una perspectiva funcional para estudiar la sociedad peruana, difundía la imagen teórica y formal de un sistema que desarrolla una tendencia a la estabilidad y orden vigente. La investigación se preocupaba por develar la temática de la integración social y de los problemas sociales: "el obrero industrial", "la prostitución", "las pautas de migración", etc. En esta etapa, la sociología se caracteriza por exponer un hondo "desencuentro entre sociedad y la ciencia social", 7 no era capaz de acercarse al estudio de la realidad nacional peruana y proponer una interpretación que dé cuenta de las grandes movilizaciones campesinas, del sindicalismo clasista, la radicalización estudiantil y el crecimiento de la izquierda peruana que sacudían el país por esos años. No se trataba de que el cambio social y la investigación empírica estuvieran ausentes en la sociología, sino más bien respondía a una perspectiva teórica vinculada al estructural-funcionalismo que imposibilitaba encontrar explicaciones profundas en la propia realidad nacional. Una vez más la sociología se encontraba atrapada en una concepción eurocéntrica, que apelaba a conceptos y modelos desarrollados en otras latitudes.

Es precisamente Bourricaud quien publica uno de los libros más importantes de la sociología peruana de esa etapa, Poder y sociedad en el Perú contemporáneo (1967), donde desarrolla un estudio de los grupos sociales y partidos políticos del país, y destaca la flexibilidad de la oligarquía para integrar los procesos de movilización de los sectores marginados de la sociedad. 
Sin embargo, el divorcio de la sociología con la realidad llevó a ensayar otra forma de entender la realidad social. Es Aníbal Quijano quien inicia tempranamente la crítica al estructural-funcionalismo en un artículo sobre Wright Mills (1963) y en Imagen y tareas del sociólogo en la sociedad peruana (1966). En su tesis doctoral, La emergencia del grupo cholo y sus implicancias en la sociedad peruana (1964), desarrolla una abierta polémica con la teoría "funcionalista" y en una perspectiva impregnada de "izquierda" -como él mismo lo señalara-, propone la especificidad de la realidad peruana y latinoamericana, al interpretar el proceso de surgimiento de un nuevo sector social y cultural en la sociedad peruana. La novedad del trabajo radica cuando sugiere los conceptos de dependencia y heterogeneidad estructural, puntos centrales en la renovación y en el debate teórico de la sociología en América Latina de los siguientes años.

En ese sentido, Aníbal Quijano, en su trabajo Naturaleza, situación y tendencia de la sociedad peruana actual (1967), y Julio Cotler, en su ensayo Mecánica de la dominación tradicional y el cambio social en el Perú (1968), iniciaron, a mitad de la década del sesenta, el estudio sobre el conflicto y el ejercicio del poder como elementos articuladores de la sociedad peruana, dentro de la perspectiva de un estudio integral y totalizador. Proceso que respondía al desarrollo de una determinada tendencia en América Latina, la sociología como disciplina experimentaba una expansión más original con la influencia de la teoría de la dependencia y el desarrollo (Fernando Henrique Cardoso, Enzo Faletto, Ruy Mauro Marini, Pablo Gonzales Casanova, Teotonio Dos Santos, entre otros).

La sociología en el Perú se institucionaliza en un debate abierto entre perspectivas diferentes, el estructural-funcionalismo y la teoría marxista (y la teoría de la dependencia). Sin embargo, ambas corrientes eran parte de un mismo proceso, la sociología fue consecuencia de un impulso significativo del desarrollo de una mentalidad abierta, basada en perspectivas teóricas modernas, en una investigación empírica y un rechazo a las posiciones sustentadas en ensayos especulativos y carentes de bases empíricas. 


\section{Sociología, Poder y revolución}

Durante el gobierno militar (1968-1980), las relaciones entre sociedad y sociología tienen una significación mayor. En un contexto de profundos cambios y de cancelación del orden oligárquico, impulsados por el régimen velasquista, es cuando la sociología asume una "identidad revolucionaria" en el Perú. ${ }^{8}$

En la Universidad de San Marcos, el movimiento estudiantil asume posiciones cada vez más radicales de izquierda y presiona por desarrollar una sociología de orientación claramente marxista. Un proceso similar, aunque en menor medida, se desarrolla en la Universidad Católica con el desplazamiento de los profesores extranjeros, quienes son reemplazados por nacionales. La sociología asume la perspectiva del poder como elemento central para estudiar la sociedad peruana, privilegiando un enfoque macro y de interpretación global. La sociología se identifica con "ciencia social", siendo capaz de incursionar en cualquier problemática de la sociedad y que haga posible entender las reformas estructurales del país. ${ }^{9}$

Las necesidades del gobierno reformista de Velasco trajeron consigo una brusca ampliación de los aparatos de Estado peruano y con ello la incorporación significativa de profesionales de la sociología. El resultado fue que el mercado de trabajo del sociólogo aumentó considerablemente, creció el número de Escuelas de Sociología y, por consiguiente, se amplió la cantidad de alumnos matriculados y los profesores de la disciplina. En ese período, esa tendencia de la sociología desarrolla una perspectiva muy integrada al Estado peruano de entonces, estaba relacionada a la elaboración ideológica del modelo político del gobierno de Velasco como una "democracia de participación plena", al desarrollo del trabajo de 
promoción y organización social de la población, como promotores, comunicadores, concientizadores, etc., y en los equipos de investigación para la planificación de las reformas sociales. Fue Carlos Delgado, en Testimonio y lucha (1973), quien argumenta mejor a favor de una "sociología del proceso revolucionario de la Fuerza Armada".

Otra tendencia de la sociología persistió en una posición crítica, desde puntos de vista más contestatarios, radicalizó el discurso y con alternativas que pretendían desarrollar formas sociales de cambio genuinamente revolucionarios desde la propia población. Una tendencia minoritaria está representada por la revista Sociedad y Política (editada por Aníbal Quijano, César Germaná, Julio Cotler y Rodrigo Montoya), que buscó desarrollar un marxismo abierto, basado en la investigación de la realidad nacional y en la reflexión teórica de América Latina, se convirtió en una de las formas de pensamiento más avanzado del continente.

Sin embargo, la tendencia mayoritaria se desarrolla en las universidades públicas con una concepción dogmática y especulativa del marxismoleninismo, alejado de toda investigación empírica de los principales problemas del país y -lo que es más importante- la argumentación se subordinaba a una concepción idealizada que tenía mucho de ideológico y valorativo. Esa sociología desarrolla fundamentalmente una imagen sustentada en un "marxismo de tipo funcionalista", los conceptos se definían en función de las clases dominantes y las estructuras de dominación, de esa manera se construye un discurso que giraba en torno a las contradicciones de la oligarquía y las clases dominadas, sometido por el imperialismo y un Estado a espaldas de la nación. ${ }^{10}$

\footnotetext{
10 Gonzalo Portocarrero: “Comentarios a 'El discurso de la Sociología en el Perú' de César Germaná", en Humberto Rodríguez y Jonny Castillo (editores): Investigaciones en ciencias sociales, un balance necesario: 1993, CONCYTEC, Lima, 1994, p. 145153.
} 
En ese contexto de debate de ideas de izquierda, se propició un cierto auge de la investigación sociológica desde mediados de la década de los setenta principalmente impulsado por centros de investigación privados, ${ }^{11}$ que tiene su mayor expresión en el libro de Julio Cotler Clases, Estado y nación en el Perú (1978), que constituye el estudio más representativo de la época. Aquí el autor desarrolla una visión integral de la formación social peruana, a partir de una reflexión de la herencia colonial y de las relaciones de dependencia. Nicolás Lynch señala que si bien el libro no formula una teoría nueva, en cambio desarrolla una síntesis del desarrollo social y político del país, lo que lo convierte en el texto más importante durante las dos décadas próximas a su edición. En esta misma línea se puede destacar el trabajo de Sinesio López El Estado oligárquico: un ensayo de interpretación (1978), quien basándose en Antonio Gramsci trata de entender la especificidad de los fenómenos políticos en el Perú.

Otros estudios desarrollados en la sociología durante los años setenta, aunque con un impacto menor que los anteriores, también merecen señalarse: dentro de la temática de la dependencia, el trabajo de Ernesto Yepes, Perú 1820-1920: un siglo de desarrollo capitalista (1974); a partir de la teoría de los modos de producción destaca el libro pionero de Rodrigo Montoya, A propósito del carácter predominantemente capitalista de la economía peruana (1970); desde la teoría de las clases sociales destacan los libros de Aníbal Quijano, Imperialismo, clases sociales y Estado en el Perú: 1890-1930 (1978), y de Henry Pease, El ocaso del poder oligárquico (1977) y Los caminos del poder (1978).

Paradójicamente la sociología peruana retrocedía a etapas oscurantistas que se creían superadas, el cultivo mayoritario volvía a un contenido claramente dogmático, especulativo y eurocéntrico del marxis- 
mo. La sociología, a partir de un pensamiento crítico más abierto, iniciaba lentamente su modificación. Se tenía que esperar a las décadas venideras para apuntar a un desarrollo más sistemático de la sociología peruana.

\section{De la revolución a la democracia y al giro constructivista}

Con la vuelta a la democracia y el proceso de desmontaje de las reformas estructurales por los gobiernos civiles en la década del ochenta se inicia una nueva etapa para el desarrollo de la sociología en el Perú. Desligada de la acción estatal, de la que gozó con el gobierno militar, la sociología inicia la búsqueda de nuevos contenidos que marcan un distanciamiento paulatino con la teoría marxista y, a la vez, permite el cultivo de prácticas de desarrollo profesional más ligados a la presencia de organizaciones no gubernamentales (ONG). Aunque la mayoría de los exponentes de la sociología seguían imprimiéndole un perfil revolucionario y marxista-leninista, especialmente en las universidades públicas, como se hizo evidente en los dos primeros congresos de sociología organizados en esos años. ${ }^{12}$

Los nuevos contenidos que va asumiendo la sociología peruana, al igual que en América Latina, se caracterizan por su evolución que discurre "de la revolución a la democracia" como eje central de la producción teórica, y por lo que se ha denominado el "giro constructivista", equivalente en ciencias sociales al "giro lingüístico" o hermenéutico de la filosofía. ${ }^{13}$

El desarrollo de la temática de la democracia significó la recuperación para la sociología del concepto weberiano de legitimidad del poder, pensamiento que dejó de lado la transformación radical de la sociedad

\footnotetext{
12 El / Congreso Peruano de Sociología organizado por la Universidad José Faustino Sánchez Carrión de la ciudad de Huacho (1981) y el Il Congreso Nacional de Sociología organizado por la Universidad Nacional de San Agustín de Arequipa (1987). 13 Emilio Lamo de Espinosa: “La sociología del siglo XX”, en REIS, № 96, 2001, p. 37.
} 
impulsada por la sociología marxista en la década pasada. La sociología evolucionaba de una visión de conflicto y propuestas de cambio a un enfoque de la "sociedad como algo dado", donde lo fundamental era la continuidad del sistema y el pacto de los actores. César Germaná describe la evolución de la sociología peruana con dos conceptos muy precisos, el paso de la "transformación radical" a la "estrategia de la concertación", ${ }^{14}$ se volvía de esta forma a las posiciones originales de nuestros primeros sociólogos de inicios del siglo XX. En esta perspectiva, y desde la denominada teoría de la transición a la democracia, se impulsa la revista "El zorro de Abajo", en la que colaboran Alberto Adrianzén, Nicolás Lynch, Sinesio López y Carlos Iván Degregori. La investigación más rigurosa que recoge estos planteamientos lo realiza Nicolás Lynch, a fines de los años ochenta, como parte de su tesis doctoral La transición conservadora. Movimiento social y democracia en el Perú, 1975-1978 (1992).

El giro constructivista en la sociología peruana definía que lo central no es cómo son los hechos sociales objetivos, sino cómo son interpretados o construidos por los sujetos. La sociología se reorienta hacia La construcción social de la realidad, título del libro de Berger y Luckmann (1966) que califica en toda su plenitud este proceso. Guillermo Nugent para el Perú, lo denomina como el desarrollo del "paradigma del mundo de la vida". ${ }^{15}$ El método cualitativo, en las dos últimas décadas, ha ido experimentando un progresivo desarrollo en la sociología y las ciencias sociales en general. Se empieza la revalorización del ser humano concreto como objeto central de análisis, en contraste a las excesivas abstracciones y a la deshumanización del cientificismo positivista de períodos anteriores. El

\footnotetext{
14 César Germaná: "El discurso de la Sociología en el Perú. De las certidumbres de los setenta a las dudas de los años noventa", ob. cit.

15 Guillermo Nugent: “Las perspectivas del mundo de la vida en las investigaciones de las ciencias sociales", en Debates en Sociología, № 16, PUCP, 1991.
} 
desarrollo de la sociología se caracteriza por poner énfasis en un particular estilo de estudio y entendimiento de la experiencia humana, estilo que aboga por acceder a los hombres y mujeres concretos. Lo central en la investigación es observar la realidad social "desde el punto de vista del actor", sin dejar de lado la estructura social en la que se encuentra inmerso.

Se trata del desarrollo del método cualitativo basado en informes biográficos que reflejan la trayectoria humana y de la subjetividad, así como de las acciones y reacciones del sujeto en el contexto de la vida social. La bibliografía sociológica se ha inclinado por los registros biográficos, denominados testimonios o historias de vida, obtenidos por entrevista. Giro constructivista que introdujo formas más flexibles de pensar la realidad social e incentivó a revisar nuevas problemáticas, desarrollando nuevos campos académicos en la sociología peruana como sociedad civil, vida cotidiana, movimientos sociales y mentalidades, que son parte de un mayor desarrollo en los años siguientes.

El repliegue de la sociología de la acción directa del Estado, permitió el desarrollo de la actuación de los sociólogos en las ONG, instituciones que se desempeñan en segmentos parciales de la población, con un carácter bastante pragmático y que conciben la labor fundamental de la disciplina como ingeniería social. De esta forma se refuerza la pérdida de la posición crítica de la sociología y se afianza su compromiso con una perspectiva que pierde autonomía con la práctica y la crítica social.

\section{Sociología, ciencia y profesión}

El giro constructivista y el tránsito de la "revolución a la democracia" aparecen claramente definidos por una profunda crisis de las ciencias sociales en general y de la sociología en particular. Notas sobre los problemas de la investigación social en América Latina (1990), de Aníbal Quijano, 
es el artículo que analiza la crisis de paradigmas y la crisis de problemática en la sociología, empieza una profunda revisión del carácter de la disciplina y va a influir decisivamente en el estado de incertidumbre generado, iniciando el camino de propuestas más originales en la producción científica. El predominio mayoritario del modelo marxista-leninista en la sociología peruana se quiebra en los años noventa, en gran parte por el fracaso de Izquierda Unida, la derrota de Sendero Luminoso y el desarrollo de la hegemonía ideológica del neoliberalismo, que lleva a la intelectualidad peruana a romper con la "identidad revolucionaría de la sociología".

La incertidumbre en la sociología peruana se manifiesta en todas sus dimensiones. Confusión y pluralidad de orientaciones, escuelas y estilos de conocimientos, que repercuten directamente en las temáticas de indagación y estudio de la realidad peruana, denominado con acierto como un momento de "perplejidad" teórica. Inseguridad frente a la reducción del mercado de trabajo, principalmente constreñido a algunas universidades públicas y al campo de las ONG; luego del auge de la sociología de las décadas pasadas se asiste a la desaparición de las escuelas en las universidades privadas de Lima. ${ }^{16}$ Impotencia para dar cuenta y procurar explicaciones de las transformaciones radicales que venían ocurriendo en la sociedad peruana. Este momento de incertidumbre ha tenido su mayor expresión en el debate sobre la sociología como ciencia y profesión, la "sociología aplicada" que se orienta a desarrollar una visión tecnocrática de la vida social como "ingeniero social", dispuesto a intervenir cuando el mercado lo demande, mientras que la "sociología crítica" busca la comprensión de la vida social para poner en evidencia los resortes de la dominación y el poder en una sociedad donde prima la desigualdad y

16 Se cierra la carrera de sociología en la Universidad Nacional Agraria, en la Universidad Femenina a inicios de los noventa, en la Universidad San Martín en 1997 y en la Universidad Garcilaso de la Vega, recientemente. 
explotación. Un debate desarrollado con mucha fuerza en el III Congreso Nacional de Sociología, ${ }^{17}$ en la polémica entre César Germaná de la Universidad de San Marcos y Guillermo Rochabrún de la Universidad Católica y formulada de manera concreta en las reformas curriculares de los planes de estudio de las diferentes escuelas del país en los años noventa (cuadro 1).

Cuadro 1 - Objetivos de las reformas de los planes de estudio de las especialidades de sociología

\section{Pontificia Universidad Católica del Perú, 1995-1996}

Flexibilizar el plan de estudios aumentando el número de cursos electivos. Incorporar nuevos temas y enfoques.

Consolidar la perspectiva profesional de los estudios mediante cursos instrumentales.

\section{Universidad Nacional Federico Villarreal, 1996}

Profesionalización de la Sociología mediante la introducción de cursos ad-hoc y de prácticas preprofesionales. Mayor vinculación con la realidad. Especialización en cuatro áreas: promoción social, planificación estratégica, ecología social e investigación social.

Universidad Nacional Mayor de San Marcos, 1996-1997

Profesionalización.

Renovación conceptual.

\section{Universidad Nacional Pedro Ruiz Gallo, 1993}

Profesionalización.

Especialización y actualización.

\section{Universidad Nacional San Agustín de Arequipa, 1996-1997}

Introducción de nuevos temas.

Profesionalización.

FUENTE: Portocarrero, Gonzalo y Chávez, Carmela: Enseñanza de sociología en el Perú. Un estudio de casos. Consorcio de Investigación Económico y Social - PUCP, Lima, 2001, p. 21.

17 César Germaná: “Las sociología como ciencia y como profesión”, en Sociología. III Congreso Nacional, Universidad Nacional de educación - Universidad Nacional de Cajamarca, Chosica, 1998. 
El estudio sobre la crisis del conocimiento social también trajo una profunda reflexión en el debate e investigación sociológica latinoamericana y peruana, aunque reducida a círculos académicos minoritarios. Desde América Latina la crisis del conocimiento social se plantea como una crisis de la propia subjetividad positivista moderna, desatada como parte de la mutación de todo un período histórico: aquel asociado a la modernidad europea, cuyo agotamiento envuelve también los fundamentos epistemológicos que sustentaron los modelos de conocimiento europeos impuestos en todo el mundo desde el siglo XVI. La búsqueda de una forma distinta de conocimiento, centrada en un proceso enteramente nuevo de conocer, de reencuentro y reapropiación de los saberes múltiples de América Latina, que de alguna forma originaron la utopía de una racionalidad liberadora es uno de los retos mayores de esta parte del continente. Las raíces de la crisis del conocimiento en América Latina se explican por los conceptos de eurocentrismo y la colonialidad del saber que se vienen discutiendo con mucha profundidad. La trayectoria histórica de las ciencias sociales en América Latina ha sido principalmente eurocéntrica. Tanto el origen de estas disciplinas como la mayor producción teórica se localiza en Europa y Estados Unidos. El eurocentrismo consiste en la forma de comprender la realidad de América Latina según las características y desarrollo particular de Europa. Es una perspectiva del conocimiento que se elabora desde el siglo XVII sobre los fundamentos de la colonización mundial. La respuesta a la crisis del conocimiento en América Latina se plantea desde los propios fundamentos epistemológicos de las ciencias sociales y del desarrollo de un pensamiento propio de América Latina que apunta a la descolonización del saber.

Como parte de la discusión que generó la publicación del trabajo de Aníbal Quijano y con el propósito de encontrar caminos más propios para el conocimiento social, se impulsó el desarrollo de la investigación 
metodológica en la sociología peruana, tuvo a Julio Mejía como uno de sus exponentes principales (cuadro 2). Mejía hace un estudio de la investigación social desde la perspectiva metodológica con la finalidad de reflexionar sobre los problemas actuales y perspectivas de la sociología. Los estudios sociales muestran un divorcio alarmante entre teoría y metodología, resultado, por un lado, de un desconocimiento de los métodos modernos de análisis y, por otro lado, como consecuencia de la debilidad teórica en la mayoría de las investigaciones, los paradigmas aparecen de manera externa al estudio de los datos. Las investigaciones más difundidas son las empíricas, la realidad se conceptualiza principalmente a partir de las clasificaciones de datos y constatación de sus vínculos. La teoría social pasa a un segundo plano. La dicotomía entre investigación y teoría tiene su raíz en la concepción de la teoría como un nivel separado, cuyo fin se agota en sí misma, y de la investigación como actividad principalmente de manipulación técnica de los datos. La dinámica de este ciclo de investigaciones metodológicas en la sociología de San Marcos se expresa en la publicación del libro Problemas metodológicos de las ciencias sociales en el Perú (2002). Se examina la investigación sociológica en sus dimensiones epistemológica, metodológica y técnica. La investigación sociológica se aborda como parte de la discusión teórica de las diversas perspectivas pospositivistas.

En términos más concretos, la investigación en la sociología peruana de los noventa discurrió por caminos trazados inicialmente en la etapa anterior. Se desarrolla el estudio de las instituciones y cultura política. Es importante señalar el trabajo de Romeo Grompone, Las nuevas reglas de juego. Transformaciones sociales, culturales y políticas en Lima (1999), sobre cultura política; en el campo de las instituciones destaca Nicolás Lynch, Una tragedia sin héroes. La derrota de los partidos y el origen de los independientes, Perú 1980-1992 (1999); y desde un punto de vista más teórico sobre el concepto de democracia el libro de Carlos Franco, Acerca del modo de pensar la democracia en América Latina (1998). El libro más 
Cuadro 2 - Artículos metodológicos en revistas de la Facultad de Ciencias Sociales de la Universidad de San Marcos. 1990-2002

\begin{tabular}{|c|c|c|c|}
\hline ÁREA & AUTOR & TÍTULO & $\mathrm{N}^{\circ} / \mathrm{ANO}$ \\
\hline $\begin{array}{l}\text { Epistemologia } \\
\text { Revista de } \\
\text { Sociologia }\end{array}$ & $\begin{array}{l}\text { 1. A. Quijano } \\
\text { 2. C. Germaná }\end{array}$ & $\begin{array}{c}\text { Notas sobre los problemas de la } \\
\text { Las exigencias actuales del oficio del } \\
\text { sociólogo }\end{array}$ & $\begin{array}{l}7 / 1990 \\
10 / 1996\end{array}$ \\
\hline $\begin{array}{c}\text { Investigaciones } \\
\text { Sociales }\end{array}$ & $\begin{array}{l}\text { 3. A. Scribano } \\
\text { 4. T. Molinari }\end{array}$ & $\begin{array}{c}\text { Algunas notas sobre problemas } \\
\text { epistemológicos de la investigación en } \\
\text { ciencias sociales } \\
\text { Pautas teóricas integradas en la } \\
\text { investigación: explorando identidades } \\
\text { políticas de los jóvenes en Lima }\end{array}$ & $\begin{array}{l}9 / 2002 \\
9 / 2002\end{array}$ \\
\hline $\begin{array}{l}\text { Metodologia } \\
\text { Revista de } \\
\text { Sociologia }\end{array}$ & 1. J.M ejía & $\begin{array}{c}\text { Algunos problemas metodológicos de la } \\
\text { sociología en el Perú } \\
\text { La investigación cuantitativa en la } \\
\text { sociología peruana }\end{array}$ & $\begin{array}{l}10 / 1996 \\
12 / 1999\end{array}$ \\
\hline $\begin{array}{l}\text { Investigaciones } \\
\text { Sociales }\end{array}$ & 3. S. López & $\begin{array}{c}\text { Problemas y alternativas la investigación } \\
\text { en sociología }\end{array}$ & $4 / 1999$ \\
\hline $\begin{array}{c}\text { Técnicas } \\
\text { Investigaciones } \\
\text { Sociales }\end{array}$ & 2. J.M ejía & $\begin{array}{c}\text { Técnicas cualitativas de investigación en } \\
\text { las ciencias sociales } \\
\text { El muestreo en la investigación } \\
\text { cualitativa }\end{array}$ & $\begin{array}{l}3 / 1999 \\
5 / 2000\end{array}$ \\
\hline
\end{tabular}

FUENTE: "Enseñanza de la metodología en la escuela de Sociología de la Universidad de San Marcos". En Investigaciones Sociales, № 8, UNMSM, Lima, 2000, p. 229.

importante de esta etapa de la sociología, por la diversidad de fuentes utilizadas y la riqueza de la argumentación teórica, es el de Sinesio López, Ciudadanos reales e imaginarios (1997), quien nos presenta una interpretación del Estado y la ciudadanía desde una perspectiva sociohistórica del Perú.

El afianzamiento del giro constructivista en la sociología peruana representó el impulso de las siguientes líneas de investigación: en 
movimientos sociales destaca el trabajo de Luis Pásara: La otra cara de la luna. Nuevos actores sociales en el Perú, (1991); en la temática de identidades, la ciudadanía popular es estudiada por Carlos Iván Degregori, Cecilia Blondet y Nicolás Lynch: De invasores a ciudadanos en San Martín de Porres. Conquistadores de un nuevo mundo (1986); el perfil del industrial informal por Norma Adams y Néstor Valdivia: Los otros empresarios. Ética de migrantes y formación de empresas en Lima (1991); la problemática de la identidad femenina por Norma Fuller: Dilemas de la femineidad. Mujeres de clase media en el Perú, (1993); en el campo de la mentalidad popular destacan los trabajos de Gonzalo Portocarrero: El Perú desde la escuela, (1989) y Sacaojos, crisis social y fantasmas coloniales (1990); la violencia social es abordada en toda su plenitud también por Gonzalo Portocarrero en Razones de sangre aproximaciones a la violencia política (1998); y la temática democracia y cultura política es analizada por Nicolás Lynch en Transición conservadora. Movimiento social y democracia en el Perú, 1975-1978 (1992), e Imelda Vega-Centeno en sus libros Aprismo Popular. Cultura, religión y política, (1991) y Simbólica y política: Perú 1978-1993 (1994).

En general, durante esta etapa la sociología produjo una sustitución temática y analítica que redujo la capacidad totalizadora de su análisis, desapareció la posibilidad de las ciencias sociales de establecer una explicación global de la sociedad, una visión integral que permita una interpretación de conjunto, de su desarrollo y naturaleza, lo que sí se ha conseguido es el estudio de un buen número de procesos específicos. No tenemos una visión global de país, a diferencia del sesenta y setenta que produjeron los trabajos de Bourricaud, Aníbal Quijano y Julio Cotler; lo que se ha desarrollado en la actualidad son estudios notables, aunque parciales, sobre algunos aspectos de la realidad política y social del país. Las ciencias sociales han avanzado en sus logros, en el estudio de fenómenos parciales y particulares. De la sociedad en su conjunto aún sabemos poco, cosas puntuales, aunque las ciencias sociales muestran un desarrollo sostenido, sigue siendo desigual. 


\section{Posibilidades del presente}

El inicio de un nuevo milenio manifiesta de manera transparente las grandes inquietudes que atraviesan y definen la construcción de la sociología peruana. El análisis de la producción del conocimiento social de estos primeros años muestra las posibilidades de la sociología peruana en los próximos años.

En efecto, una cuestión sustantiva de la constitución de la sociología actual es la necesidad de integración frente al proceso de división y subdivisiones que la ha dominado en el siglo XX. Movimiento de convergencia que posibilite una nueva síntesis de la estructura del conocimiento social sobre las bases de una explicación simultánea entre el cambio histórico y estructural. Proceso que ha sido reclamado constantemente por César Germaná en su libro La racionalidad en las ciencias sociales (2002) y ha tenido un impulso fundamental con la visita de Inmanuel Wallerstein a la Universidad de San Marcos (2004). Proceso de desarrollo centrípeto que afecta a todas las ramas de la ciencia. Surgen en el campo de las ciencias naturales las llamadas ciencias de la complejidad y en las humanidades aparecen los estudios culturales, movimientos que inciden en la dirección de una unificación e enriquecimiento teórico y metodológico múltiple con las ciencias sociales.

Otro aspecto imprescindible, relacionado a lo anterior, consiste en la discusión y redefinición de la identidad epistemológica de las ciencias sociales que permita sentar las bases de un nuevo paradigma que haga más comprensible el mundo. Las ciencias sociales tienen grandes limitaciones en sus premisas que ya no corresponden a la problemática de la sociedad del siglo XXI. Es importante señalar los esfuerzos realizados por Manuel Castillo en La razón del vacío. Epistemología, globalización y saber social (2001) para introducir en el debate peruano las teorías posmodernas. Por mi parte, trato de reflexionar sobre los aportes de Niklas Luhmann en la metodología en la Perspectiva de la investigación de segundo orden (2004) 
y los alcances siempre presentes de Aníbal Quijano.

El desarrollo de espacios de debate en la sociología, pese a la escasez de recursos, en particular por la mayor debilidad del Estado en la universidad pública, que prácticamente ha conllevado a una desaparición de la investigación básica, se mantiene la necesidad de una ciencia social que responda a la reconstrucción de la sociedad peruana. La existencia de revistas de sociología de las universidades de San Marcos, Arequipa, Puno, Chiclayo y Católica de Lima; el desarrollo sostenido de los Congresos de Sociología, desde 1995 a la fecha, son una muestra de la búsqueda de canales de debate e institucionalización. ${ }^{18}$ Asimismo, es importante anotar el cambio de mentalidad en los profesionales y docentes de sociología, que han dejado atrás el dogmatismo, ahora se comparte más una apertura de renovación y un propósito por actualizar su preparación académica. ${ }^{19}$ En esa misma dirección ha sido fundamental el establecimiento de estudios de post-grado en sociología y el doctorado en ciencias sociales en la Universidad de San Marcos que ha mostrado que los intelectuales peruanos reclaman una presencia científica y profesionalización cualificada.

La necesidad de un debate contemporáneo en la sociología peruana ha llevado a estudiar las grandes transformaciones globales de nuestro tiempo y sus repercusiones en la naturaleza del país. Hay una mayor conciencia de que la sociedad moderna caduca y muchas evidencias señalan que estamos en un período de transición en el cual algo está terminando y algo nuevo empieza a conformarse. América Latina y el Perú tienen que analizarse como realidades propias que se construyen dentro de un marco cada vez más mundializado. En ese sentido, aunque desde

\footnotetext{
18 El III Congreso Nacional de Sociología (1995) fue organizado por la Universidad Nacional de Cajamarca, el IV Congreso (1998) por la Universidad Nacional Federico Villarreal, el V Congreso (2002) por la Universidad Nacional del Antiplano de Puno y el VI Congreso Nacional (2004) por la Universidad Nacional del Centro, de Huancayo.

19 Gonzalo Portocarrero y Carmela Chávez: Enseñanza de sociología en el Perú. Un estudio de casos, Consorcio de Investigación Económico y Social, PUCP, Lima, 2001, p. 18.
} 
posiciones minoritarias, se generan nuevos ámbitos de desarrollo en la sociología peruana actual, que no dejan de ser fundamentales en las perspectivas futuras de la disciplina como ciencia.

Se destacan el interés por estudiar las transformaciones globales, aquí merece mención especial el esfuerzo que despliega Teivo Teivainen que dirige el "Programa de estudios sobre democracia y transformación global" en la Universidad de San Marcos, su libro Pedagogía del poder mundial. Relaciones y lecciones del desarrollo en América Latina (2003) es una muestra de la convergencia de reflexión crítica e investigación que ha logrado generar. Asimismo, el impacto de los procesos de virtualización y la informática en las relaciones sociales ha sido expuesto rigurosamente en una investigación empírica para el caso de la juventud limeña por María Teresa Quiroz en El impacto de las tecnologías del conocimiento y la comunicación en el pensar-sentir de los jóvenes (2004).

En esa misma dirección, la temática de la descolonización del saber, de modo concreto en el Perú y América Latina, ha sido abordada por Carolina Ortiz en Procesos de descolonización del imaginario y del conocimiento. Poéticas de la violencia y de la crisis (2003), en la búsqueda de formas de superación del paradigma positivista y del eurocentrismo de manera particular.

Aníbal Quijano, en sus trabajos Colonialidad del poder y clasificación $\operatorname{social}^{20}$ (2000) y, más recientemente, en Notas sobre la 'raza' y democracia en los países andinos (2003), desarrolla la temática de la identidad y el mestizaje punto central en la comprensión de la formación de la ciudadanía y constitución del Estado-nación, un viejo tema que se retoma en relación a la perspectiva teórica de la colonialidad del poder y el eurocentrismo, aspectos esenciales del conflicto crucial en la subjetividad de los países del 
Perú y Ecuador y Bolivia.

La ciudad como centro de las transformaciones son abordados por Julio Calderón, La ciudad ilegal en el siglo XX (2004), y Joseph James, Los procesos de democratización y desarrollo en escenarios meso en la megaciudad de Lima (2004), recogen esfuerzos de una visión integral del desarrollo de la ciudad de Lima, en un proceso de relaciones de grupos sociales y el conjunto de intereses que se articulan alrededor del mercado y de la intervención del Estado. En general, son trabajos que recopilan una abundante información empírica, que permite la formulación de sugerentes ideas y propuestas sobre la dinámica de la ciudad de Lima. En ese mismo sentido, como parte de las modificaciones sociales en la ciudad, el fenómeno de la violencia urbana se revela como una problemática constitutiva en los países pobres, en particular se destaca el trabajo de Martín Santos, La vergüenza de los pandilleros (2002), que establece la vigencia siempre presente de este tema en la sociología peruana. Otro aspecto central, con relación a los grandes cambios que afectan a la sociedad peruana, tiene que ver con el surgimiento de nuevos actores en la escena social, en particular de la emergencia de nuevas clases medias, desde los antiguas invasiones y asentamientos humanos y como parte del proceso de mestizaje que se consolida e irrumpe modificando drásticamente las fuerzas sociales, el consumo y la cultura del país, este proceso es analizado en el libro Perú hoy. La clase media iexiste? (2003).

Un hecho que ha definido a la sociedad peruana en los últimos años es la proliferación de la crueldad, el cinismo, la corrupción y las formas de adicción, denominado como la sociología del mal y desde una aproximación con las humanidades, especialmente con la filosofía, literatura y el psicoanálisis, permiten a Gonzalo Portocarrero en su libro Rostros criollos del mal (2004) abrir una temática que por su riqueza de análisis y propuestas explicativas definirá uno de los campos más singulares de la sociología peruana. 
Quizás lo que mejor caracteriza el inicio de un nuevo milenio en la sociología es la recuperación de la problemática del desarrollo desde una perspectiva latinoamericana. Aníbal Quijano, en El laberinto de América Latina: ¿hay otras salidas? (2004), ha señalado que después de décadas de pérdida de confianza en sus posibilidades, se asiste a un debate sobre el desarrollo como resultado de la deslegitimación del neoliberalismo y por el protagonismo del desarrollo de un capitalismo nacional en el Brasil, el afianzamiento del liberalismo en países como el Perú y las posibilidades de encontrar vías más originales y equitativas por el Foro Social Mundial. Sin duda, es uno de los campos más importantes de la sociología peruana futura.

En fin, el cambio del milenio en la sociología peruana, a pesar de las limitaciones institucionales, permite avizorar nuevos contenidos y campos de progreso. En especial destacan la necesidad de reunificarse como una disciplina totalizadora que se aproxima más a las humanidades, la urgencia de estudiar las implicancias de las transformaciones globales en la realidad nacional y el ímpetu por discutir su identidad epistemológica. La sociología marcha hacia la exigencia de construir un paradigma endógeno, que no sólo se encuentre enraizado en nuestras propias circunstancias, sino que también, exprese la complejidad de la realidad. Todo esto hace pensar que la sociología peruana tiene un desarrollo garantizado.

\section{Referências}

ADAMS, Norma y VALDIVIA, Néstor. Los otros empresarios. Ética de migrantes y formación de empresas en Lima. Lima: IEP, 1991.

BELAUNDE, Víctor Andrés. La realidad nacional. París: editora, 1931.

BOURRICAUD, Francois. Poder y Sociedad en el Perú. Lima: IEP, 1989.

BRUNNER, José Joaquín. Sobre el crepúsculo de la sociología y el comienza de otras narrativas. Sociedad hoy, revista de Ciencias Sociales, vol. 1, n. 1, año 1. 1997. 
CALDERÓN, Julio. La ciudad ilegal en el siglo XX. Tesis Doctoral. Lima: UNMSM, 2004.

COTLER, Julio. Algunas reflexiones sobre el futuro de las ciencias sociales en América Latina. Columbia University, Papers on Latin America, № 9. 1989.

COTLER, Julio. Clases, Estado y Nación en el Perú. Lima: IEP, 1978.

COTLER, Julio. La mecánica de la dominación interna y del cambio social. In: Perú Problema. Cinco Ensayos. Perú Problema 1. Lima: IEP, 1968.

DEGREGORI, Carlos Iván. El surgimiento de Sendero Luminoso. Lima: IEP, 1990.

DEGREGORI, Carlos Iván. Sendero Luminoso: los hondos y mortales desencuentros. Lima: IEP, 1985.

DEGREGORI, Carlos Iván; BLONDET, Cecilia y LYNCH Nicolás. De invasores a ciudadanos en san Martín de Porres. Conquistadores de un nuevo mundo. Lima: Lima, 1986.

DELGADO, Carlos. Testimonio de Lucha. Lima: Ediciones Peisa, 1973.

Del CASTILLO, Daniel. La autobiografía en el Perú contemporáneo. MARGENES, Lima, n. 12, SUR Casa de estudios del Socialismo. 1994.

FULLER, Norma. Dilemas de la femineidad. Mujeres de clase media en el Perú. Lima: PUCP, 1993.

FLORES GALINDO, Alberto. Buscando un Inca. Identidad y utopía en los andes. Lima: Horizonte, 1988.

FRANCO, Carlos. Acerca del modo de pensar la democracia en América Latina. Lima: Fundación Friedrich Ebert, 1998.

FRANCO, Carlos. La otra modernidad. Imágenes de la sociedad peruana. Lima: CEDEP, 1991.

JAMES, Joseph. Los procesos de democratización y desarrollo en escenarios meso en la megaciudad de Lima. Tesis Doctoral. Lima: UNMSM, 2004.

GARCÍA CALDERÓN, Francisco. El Perú contemporáneo. Lima: Fondo Editorial del Congreso del Perú, 2001. 
GERMANÁ, César. La racionalidad en las ciencias sociales. Lima: Fondo editorial de la Facultad de Ciencias Sociales, UNMSM, 2002.

GERMANÁ, César. Las exigencias actuales del oficio del sociólogo. Revista de Sociología, Lima, no 10, UNMSM, 1996.

GERMANÁ, César. La sociología como ciencia y como profesión. Debates en Sociología, Lima, n. 20-21. Departamento de Ciencias Sociales. Pontificia Universidad Católica del Perú. 1998.

GERMANÁ, César. Las sociología como ciencia y como profesión. In: Sociología. III Congreso Nacional, Universidad Nacional de educación - Universidad Nacional de Cajamarca, Chosica. 1998.

GERMANÁ, César. El discurso de la sociología en el Perú. De las certidumbres de los años setentas a las dudas de los años noventas. In: Investigaciones en Ciencias Sociales, un balance necesario: 1993. Lima: CONCYTEC, 1994.

GERMANÁ, César. El análisis sociológico del proceso político y del Estado en el Perú: un balance de la sociología política. I Congreso Peruano de Sociología, Huacho. 1982.

GERMANÁ, César y LOAYZA, Sulema. La enseñanza de las Ciencias Sociales a nivel introductorio en la Universidad de San Marcos. Universidad y Sociedad, Lima, n. 6. 1998.

GIDDENS, Anthony. En defensa de la sociología. Madrid: Alianza Editorial, 2000. GONZÁLES PRADA, Manuel. Páginas Libres. Lima: Editorial Universo, 1976.

GROMPONE, Romeo. Las nuevas reglas de juego. Transformaciones sociales, culturales y políticas en Lima. Lima: IEP, 1999.

GROMPONE, Romeo. El velero en el viento. Lima: IEP, 1991.

HERNÁNDEZ, Max y otros. Entre el mito y la Historia. Psicoanálisis y pasado andino. Lima: Ediciones Psicomalíticas Imago, 1987.

HAYA DE LA TORRE, Víctor Raúl. El antiimperialismo y el APRA. Lima: Amauta, 1972.

LAMO DE ESPINOSA, Emilio. La sociología del siglo XX. Revista Española de Investigaciones Sociológicas, Madrid, n. 96. 2001. 
LANDER, Edgardo (Org.). La colonialidad del saber: eurocentrismo y ciencias sociales. Perspectivas latinoamericanas. Buenos Aires: CLACSO - UNESCO, 2000.

LECHNER, Norbert. Las transformaciones de la política. Revista Mexicana de Sociología. Año LVIII, n.1. Enero-marzo. 1996.

LÓPEZ, Sinesio. Ciudadanos reales e imaginarios. Lima: IDS, 1997.

LÓPEZ, Sinesio. Política, violencia y revolución. El Zorro de Abajo, Lima, n. 7, junio. 1987.

LÓPEZ, Sinesio. Perú 1985: entre la moderación y la radicalidad. El Zorro de Abajo, Lima, n. 1, junio-julio. 1985.

LÓPEZ, Sinesio. El Estado oligárquico: un ensayo de interpretación. Revista Mexicana de Sociología. México, UNAM, Año XL/Nol. 3, 1978.

LYNCH, Nicolás. La sociología y el estudio de la política en el Perú. Investigaciones Sociales, Lima, n. 8, UNMSM. 2001.

LYNCH, Nicolás. Una tragedia sin héroes. La derrota de los partidos y el origen de los independientes. Perú 1980-1992. Lima: Fondo Editorial de la Universidad Nacional Mayor de San Marcos, 1999.

LYNCH, Nicolás. La Transición Conservadora. Movimiento Social y Democracia en el Perú, 1975-1978. Lima: El Zorro de Abajo ediciones, 1992.

MARIÁTEGUI, José Carlos. Siete ensayos de interpretación dela realidad peruana. Lima: Editorial Amauta, 1970.

MATOS MAR, José. Desborde popular y crisis del Estado veinte años después. Lima: Fondo Editorial del Congreso del Perú, 2004.

MEJÍA, Julio. Sociedad y conocimiento en América Latina. Notas introductorias. Investigaciones Sociales, Lima, n. 12, UNMSM, 2004.

MEJÍA, Julio. Perspectiva de la investigación social de segundo orden. In: OSORIO, Francisco. Ensayos sobre socioautopoiesis y epistemología constructivista. Santiago: Ediciones MAD, Facultad de Ciencias Sociales, Universidad de Chile, 2004.

MEJÍA, Julio. Problemas metodológicos de las ciencias sociales en el Perú, Lima: Fondo Editorial de la Facultad de Ciencias Sociales UNMSM, 2002. 
MEjÍA, Julio.Enseñanza de la metodología en la escuela de Sociología de la Universidad de San Marcos. Investigaciones Sociales, Lima, n. 8, UNMSM, 2002.

MONTOYA, Rodrigo. A propósito del carácter predominantemente capitalista de la economía peruana. Lima, 1970.

NUGENT, Guillermo.Las perspectivas del mundo de la vida en las investigaciones de las ciencias sociales. Debates en Sociología, Lima, n. 16, PUCP. 1991.

NUGENT, Guillermo et al. Perú hoy. La clase media iexiste?. Lima: Desco, 2003.

ORTIZ, Carolina. Procesos de descolonización del imaginario y del conocimiento. Poéticas de la violencia y de la crisis. Tesis Doctoral. Lima: UNMSM, 2003.

PALMA, Clemente. El porvenir de las razas en el Perú. Tesis Bachiller en Letras.Lima: Imprenta Torres Aguirre,1897.

PASARA, Luis y otros. La otra cara de la luna. Nuevos actores sociales en el Perú. Buenos Aires: CEDYS, 1991.

PARODI, Jorge y TWANAMA, Walter. Los pobladores, la ciudad y la política: un estudio de actitudes" In: Los pobres, la ciudad y la política. Lima: Jorge Parodi editor. Centro de Estudios de Democracia y Sociedad, 1993.

PEASE, Henry. Los caminos del poder. Tres años en la escena política. Lima: DESCO, 1978.

PEASE, Henry. El ocaso del poder oligárquico. Lima: DESCO, 1977.

PODESTÁ, Bruno. Estado de las ciencias sociales en el Perú. Lima: Universidad del Pacífico, 1978.

PORTOCARRERO, Gonzalo. Rostros criollos del mal. Cultura y trasgresión en la sociedad peruana. Lima: Red para el desarrollo de la Ciencias Sociales en el Perú, 2004.

PORTOCARRERO, Gonzalo. Razones de sangre. Aproximaciones a la violencia política. Lima: PUCP, Fondo Editorial, 1998.

PORTOCARRERO, Gonzalo. La cuestión racial: espejismo y realidad sacaojos. In: Violencia estructural en el Perú: Sociología. Lima: APEP, 1990. 
PORTOCARRERO, Gonzalo. El Perú desde la escuela. Lima: IAA, 1989.

PORTOCARRERO, Gonzalo y CHÁVEZ, Carmela. Enseñanza de sociología en el Perú. Un estudio de casos. Lima: Consorcio de Investigación Económico y Social PUCP, 2001.

PORTOCARRERO, Felipe.El pensamiento político de Haya de la Torre. Análisis, Lima, n. 1, 1977.

QUIJANO, Aníbal. El laberinto de América Latina: ¿hay otras salidas?. Revista Venezolana de Economía y Ciencias Sociales, Caracas, vol. 10, n. 1, 2004.

QUIJANO, Aníbal. Notas sobre "raza" y democracia en los países andinos. Revista Venezolana de Economía y Ciencias Sociales, Caracas, vol. 9, n.1, 2003.

QUIJANO, Aníbal. El fantasma del desarrollo en América Latina. Revista Venezolana de Economía y Ciencias Sociales, Caracas, vol. 6, n. 2, 2000.

QUIJANO, Aníbal. Colonialidad del poder y clasificación social", Journal of WorldSystems Research, vol. XI, n. 2, University of California, <http//www.jwsr.ucr.edu>.

QUIJANO, Aníbal. Notas sobre los problemas de la investigación social en América Latina. Revista de Sociología, Lima, n. 7, Universidad Nacional Mayor de San Marcos. 1990.

QUIJANO, Aníbal. Sociedad, poder y sociología en el Perú. I Congreso Peruano de Sociología, Huacho. 1982.

QUIJANO, Aníbal. Imperialismo, clases sociales y Estado en el Perú: 1890-1930. Lima: Mosca Azul, 1978.

QUIJANO, Aníbal. Naturaleza, situación y tendencia de la sociedad peruana actual. La Habana: Pensamiento crítico, 1967.

QUIJANO, Aníbal. Imagen y tares del sociólogo en la sociedad peruana. Separata de la revista Letras, Lima, n. 74-75, UNMSM. 1966.

QUIROZ, María Teresa. El impacto de las tecnologías del conocimiento y la comunicación en el pensar - sentir de los jóvenes. Tesis Doctoral. Lima: UNMSM, 2004.

ROCHABRÚN, Guillermo. Horizontes y Discursos en la sociología peruana. In: El Perú frente al siglo XXI. Lima: Pontificia Universidad Católica del Perú, 1995. 
ROCHABRÚN, Guillermo. ¿Crisis de paradigmas o falta de rigor?. Debates en Sociología, n. 19, Lima: Pontificia Universidad Católica del Perú, 1994.

ROCHABRÚN, Guillermo. Socialidad e individualidad. Materiales para una sociología. Lima: PUCP, 1993.

ROCHABRÚN, Guillermo. Sociología y sociedad en el Perú: un esbozo histórico. In: Estudios de historia de la ciencia en el Perú. Lima: CONCYTEC - SOPHICYT, 1986.

ROCHABRÚN, Guillermo. La producción sociológica en el Perú. I Congreso Peruano de Sociología, Huacho. 1982.

SANTOS, Martín. La vergüenza de los pandilleros. Masculinidad, emociones y conflictos en esquineros del cercado de Lima. Lima: CEAPAZ, 2002.

SONNTAG, Heinz. Duda/ certeza/ crisis. La evolución de las ciencias sociales en América Latina. Caracas: UNESCO y Nueva Sociedad, 1988.

TEIVAINEN, Teivo. Pedagogía del poder mundial. Relaciones y lecciones del desarrollo en América Latina. Lima: Cedep, 2003.

TANAKA, Martín. Los espejismos de la democracia. Lima: IEP, 1998.

VEGA-CENTENO, Imelda. Simbólica y Política: Perú 1978-1993. Lima: Fundación Ebert, 1994.

VEGA-CENTENO, Imelda. Aprismo Popular. Cultura,Religión y política. Lima: PUCP - Tarea, 1991.

VERGARA, Jorge y GOMÁRIZ, Enrique. Teoría, epistemología y poder en la sociología latinoamericana de los noventa. Un análisis desde la perspectiva de su crisis teórica. FEMENTUM. Revista Venezolana de sociología y Antropología, Mérida, año 3, n. 6 y 7.1993.

WALSH, Catherine. La (re)articulación de subjetividades políticas y diferencia colonial en el Ecuador. Reflexiones sobre el capitalismo y las geopolíticas del conocimiento. In: Indisciplinar las ciencias sociales. Geopolíticas del conocimiento y colonialidad del poder. Perspectiva desde lo andino. Quito: Universidad Andina Simón Bolívar y Ediciones ABYA-YALA, 2000. 
WALLERSTEIN, Immanuel. Retos para la universidad en el siglo XXI. Investigaciones Sociales, Lima, n. 13, UNMSM, 2004.

WALLERSTEIN, Immanuel. Conocer el mundo, saber el mundo: el fin de lo aprendido. Una ciencia para el siglo XXI. México: Siglo XXI y Centro de Investigaciones Interdisciplinarias en Ciencias y Humanidades de la UNAM,2001.

WALLERSTEIN, Immanuel. Impensar las ciencias sociales. Límites de los paradigmas decimonónicos. México: Siglo XXI y Centro de Investigaciones Interdisciplinarias en Ciencias y Humanidades de la UNAM, 1999.

WALLERSTEIN, Immanuel. Abrir las ciencias sociales. México: Siglo XXI y Centro de Investigaciones Interdisciplinarias en Ciencias y Humanidades de la UNAM, 1996.

YEPES, Ernesto. Perú 1820-1920. Un siglo de desarrollo capitalista. Lima: IEP, 1972.

Recebido: 02/03/2005

Aceite final: 19/04/2003 


\section{Resumo}

El trabajo es una síntesis de la evolución de la sociología en el Perú desde su incorporación como cátedra universitaria en 1896. Se elabora un discurso que busca vincularse con los avatares propios del desarrollo de la modernidad en el país y, al mismo tiempo, se apoya en el debate teórico resultado del lento proceso de institucionalización de la disciplina y de la emergencia de una mayor conciencia, en los últimos años, acerca de la pluralidad de paradigmas, perspectivas metodológicas y posibilidades interpretativas de la realidad social.

Palabras-clave: Pensamiento social, sociología como cátedra, poder y revolución, constructivismo, institucionalización de la disciplina, racionalidad y unidisciplinariedad. 


\section{The development of sociology in Peru. Introductory notes}

\section{Julio Mejía Navarrete}

This work is a synthesis of the evolution of sociology in Peru since its incorporation as a university cathedra in 1896. A discourse is build in order to link to the changes that are typical of the developments of modernity in the country and, at the same time, it is supported on the theoretical debate resulting from the slow process of institutionalization of the discipline and the emergence of a higher awareness of the plurality of paradigms, methodological perspectives, and interpretive possibilities on social reality in recent years.

Key words: Social Thinking, Sociology as a cathedra, power and revolution, constructivism, institutionalization of the discipline, rationality and unidisciplinarity. 